\title{
Efeito da reação de calcinação nas análises energética e exergética de um forno rotativo empregado na produção de clínquer
}

\section{(Effect of the calcination reaction on the energy and exergy analysis of a rotary kiln used in the production of clinker)}

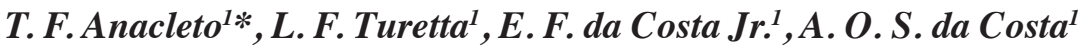 \\ ${ }^{1}$ Universidade Federal de Minas Gerais, Programas de Pós-Graduação em Engenharia Química e Engenharia \\ Mecânica, Departamento de Engenharia Química, Belo Horizonte, MG, Brasil
}

\begin{abstract}
Resumo
A produção do cimento exige um grande consumo energético. A principal etapa desse processo é a clinquerização, onde ocorre a produção do clínquer, que é realizada em forno rotativo. Estudos publicados em literatura especializada indicam que parte significativa da energia gasta no processo produtivo do cimento é utilizada nesta parte do sistema. Em vista disso, é relevante o estudo sobre a utilização da energia disponibilizada para a operação do forno rotativo. Diversos autores realizaram a modelagem matemática de fornos rotativos objetivando analisar as eficiências energética e exergética desse equipamento. Neste trabalho, partindo-se de um estudo anterior, foi proposta uma metodologia para o cálculo das eficiências energética e exergética de um forno empregado na produção do clínquer. Foram adicionadas as reações envolvidas na calcinação e clinquerização, o que tornou a descrição do processo mais realista. Essas alterações afetaram significativamente os valores das eficiências obtidas anteriormente.
\end{abstract}

Palavras-chave: análise energética, análise exergética, forno rotativo de clínquer, cimento.

Abstract

The production of cement requires high energy consumption. The main process of cement production is the clinker production called clinkerization. This process occurs in a rotary kiln. Previous studies indicate that a considerable part of the energy spent in the cement production process is used in clinker production. Thus, the study on the use of the energy available for the operation of the rotary kiln is relevant. Several authors used mathematical modeling of rotary kilns to analyze the energy and exergy efficiencies of this equipment. In this study, it was proposed a methodology to calculate the energy and exergy efficiencies of a kiln used in clinker production. The reactions involved in calcination and clinkerization were added and this modification resulted in a more realistic process description. These changes significantly affected the efficiency values previously obtained.

Keywords: exergetic analysis, energetic analysis, clinker rotary kiln, cement.

\section{INTRODUÇÃO}

A produção de cimento referente aos 20 maiores países produtores mundiais no ano de 2015 foi estimada em 3,5 bilhões de toneladas, conforme os dados mais recentes disponibilizados pela Associação Europeia de Cimento [1]. Uma produção desta magnitude exige um grande consumo energético [2]. A principal etapa na produção de cimento é a clinquerização, na qual ocorre a produção do clínquer [3]. Um estudo realizado em uma indústria cimenteira do Marrocos demonstrou que cerca de $35 \%$ da energia total fornecida à planta foi utilizada nessa etapa [4]. Além disso, com base nos dados da GNR (Getting the Numbers Right) para o ano de 2014, a demanda energética mundial média para a etapa de clinquerização foi de $3510 \mathrm{MJ} /$ ton de clínquer [5]. Em vista disso, infere-se que é relevante um estudo que analise a utilização da energia disponibilizada para este sistema.

*tulioeq@gmail.com
O forno rotativo de clínquer faz parte da unidade de piroprocessamento [3, 6-8]. Essa unidade é composta pela torre de ciclones, onde ocorre o pré-aquecimento, pelo forno e por um resfriador [8], conforme o esquema ilustrado na Fig. 1. No processo, a farinha passa inicialmente pela torre de ciclones. Nessa etapa, parte do carbonato de cálcio se decompõe por uma reação denominada calcinação. Em seguida, a farinha pré-calcinada passa pelo forno rotativo, onde ocorre o restante da calcinação e as reações de formação do clínquer. Finalmente, o clínquer formado é resfriado [9]. Neste processo, os gases de combustão fluem em contracorrente. Eles são gerados principalmente no forno e deixam o sistema pelo topo da torre de ciclones. Aplicando a primeira e a segunda leis da termodinâmica ao forno, é possível realizar uma análise energética e exergética do processo de clinquerização [11]. A análise de energia é realizada com base apenas na primeira lei da termodinâmica. Isso faz com que nessa análise as perdas de energia devido às irreversibilidades do processo sejam omitidas. Por outro lado, a análise da exergia é capaz de evidenciar a degradação 
da energia envolvida no processo quantitativamente [12]. Isso ocorre porque ela leva em consideração a união entre as duas leis enunciadas. Ou ainda, a junção do equacionamento dessas leis resulta em uma equação que possibilita explicitar a quantidade de energia que se torna indisponível para a realização de trabalho quando um determinado processo é realizado. Logo, a exergia indica o trabalho máximo que pode ser realizado empregando o processo e que, devido às inerentes irreversibilidades do sistema, se torna indisponível. A exergia é uma propriedade termodinâmica. Sua avaliação se dá tradicionalmente em relação a um estado de referência denominado frequentemente de estado morto [13]. Um sistema no estado morto está sob as mesmas temperatura e pressão do ambiente, bem como em repouso em relação ao mesmo. Dessa forma, não há interação entre sistema e ambiente.

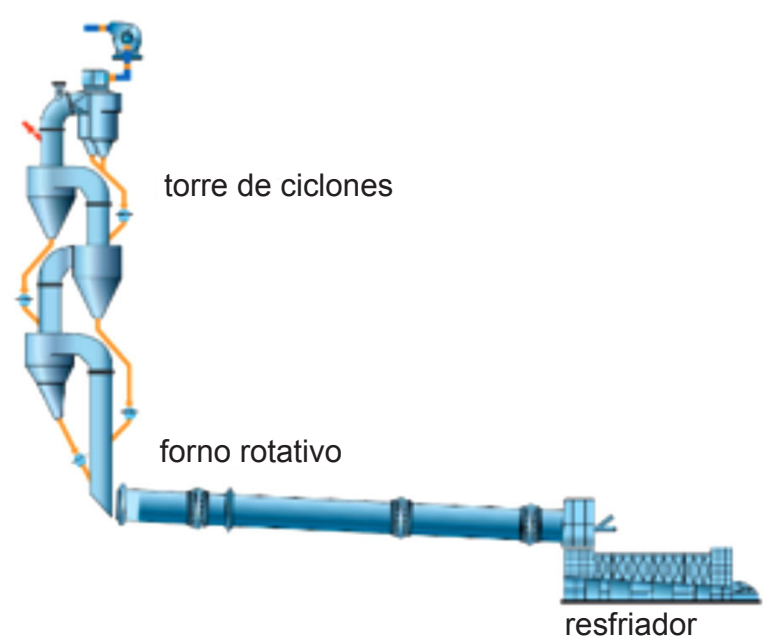

Figura 1: Unidade de piroprocessamento; adaptado de [10]. [Figure 1: Pyroprocessing unit; adapted from [10].]

Devido à importância dos fornos rotativos de clínquer na produção de cimento, diversos autores realizaram a modelagem desse equipamento com o intuito de realizar os balanços de massa, energia e exergia [10-12, 14]. A partir desses balanços, é possível calcular as eficiências energética e exergética do forno. Contudo, os autores apresentam diferentes equacionamentos para a realização dos balanços. Além disso, são realizadas diferentes considerações. Certas abordagens não consideram as reações químicas que ocorrem no forno $[11,12,14]$. Outros autores consideram apenas as reações de clinquerização [10]. Em vista disso, neste trabalho foi escolhido um estudo prévio como base. As modificações realizadas são comparadas com esse mesmo estudo. Assim, é possível se obter uma análise mais realista. Tais alterações são feitas considerando principalmente informações reportadas por profissionais da indústria. Dessa forma, o objetivo deste trabalho é realizar os cálculos das eficiências energética e exergética para um forno rotativo de clínquer, considerando os dados disponíveis e a modelagem utilizada em um estudo prévio [12]. Contudo, neste trabalho, diferente do que é reportado no estudo analisado [12], são consideradas reações envolvidas na calcinação e clinquerização. A influência da consideração de tais reações nas eficiências, especialmente a reação de calcinação, é analisada.

\section{METODOLOGIA}

Para que fosse possível analisar as eficiências energéticas e exergéticas, o volume de controle (VC) adotado para o estudo foi o forno rotativo esquematizado na Fig. 2. As correntes de entrada e saída consideradas também são esquematizadas na Fig. 2. Para a realização dos balanços de massa, energia e exergia foram adotadas as seguintes considerações: i) o sistema opera em regime permanente; ii) as variações de energia e exergia cinética e potencial são insignificantes; iii) não ocorrem variações de pressão ou de volume (fronteiras do forno rígidas); iv) não há transferência de energia na forma de trabalho através das fronteiras do sistema; v) não há exergia associada na forma de trabalho através das fronteiras do sistema; e vi) as exergias químicas específicas dos componentes foram desconsideradas. Considerou-se apenas a exergia química envolvida na reação de combustão dos componentes do combustível, contabilizada no termo da exergia de entrada. O estudo prévio [12] informa as composições, vazões mássicas e as temperaturas de cada corrente. Contudo, os valores de capacidade calorífica não são informados. Neste trabalho, foram utilizados os valores fornecidos pelo National Institute of Standards and Technology [15].

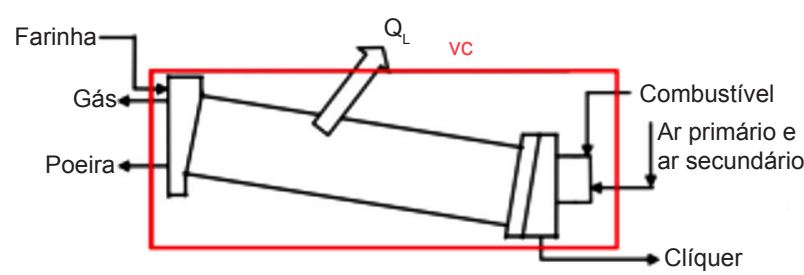

Figura 2: Volume de controle utilizado para a modelagem. [Figure 2: Control volume used for the modeling.]

Balanço de massa e energia: o balanço de massa global pode ser descrito pelas Eqs. A e B:

$$
\begin{aligned}
& \sum \mathrm{m}_{\text {entra }}=\sum \mathrm{m}_{\text {sai }} \\
& \sum \mathrm{m}_{\text {farinha }}+\sum \mathrm{m}_{\text {combustivel }}+\sum \mathrm{m}_{\text {mar }}+ \\
& \sum \mathrm{m}_{\text {clíquer }}+\sum \mathrm{m}_{\text {poeira }}+\sum \mathrm{m}_{\text {gases }}
\end{aligned}
$$

Os dados reportados da literatura referentes à composição, vazão mássica e temperatura [12] estão apresentados na Tabela AI (Apêndice). O balanço de energia para esse processo pode ser descrito pela Eq. C [13, 16-19]:

$$
\sum \mathrm{m}_{\text {entra }} \cdot \mathrm{h}_{\text {entra }}=\sum \mathrm{m}_{\text {sai }} \cdot \mathrm{h}_{\text {sai }}+\sum \mathrm{Q}
$$

Neste trabalho, a perda de calor pelas paredes do forno foi determinada pela diferença entre os outros dois termos, correspondentes à energia que entra e deixa o sistema 
em função do fluxo de massa. A eficiência energética do processo foi calculada pela Eq. D [10, 12]:

$$
\eta_{\mathrm{e}}=\frac{\left|\sum \mathrm{m}_{\text {entra }} \cdot \mathrm{h}_{\text {entra }}\right|}{\left|\sum \mathrm{m}_{\text {sai }} \cdot \mathrm{h}_{\text {sai }}\right|}
$$

Balanço de exergia: a exergia é uma propriedade termodinâmica. Assim, seu valor independe do histórico do sistema e por isso tradicionalmente os autores separam os diferentes tipos (efeitos) de exergia presentes no processo, e estes são computados separadamente. Neste estudo, a contribuição para a exergia total $\left(\varepsilon_{\text {tot }}\right)$ de um fluxo de matéria foi dividida em duas parcelas: exergia específica termomecânica $\left(\varepsilon_{\mathrm{f}}^{\mathrm{TM}}\right)$ e exergia específica química $\left(\varepsilon_{\mathrm{f}}^{\mathrm{CH}}\right)$ [11], conforme explicitado nas Eqs. E a G:

$$
\begin{aligned}
& \varepsilon_{\mathrm{tot}}=\varepsilon_{\mathrm{f}}^{\mathrm{TM}}+\varepsilon_{\mathrm{f}}^{\mathrm{CH}} \\
& \varepsilon_{\mathrm{f}}^{\mathrm{CH}}=\left(\mu_{\mathrm{i} 0}-\mu_{\mathrm{i} 00}\right) \\
& \varepsilon_{\mathrm{f}}^{\mathrm{TM}}=\varepsilon_{\mathrm{f}}^{\mathrm{PH}}+\varepsilon_{\mathrm{f}}^{\mathrm{KN}}+\varepsilon_{\mathrm{f}}^{\mathrm{PT}}=\left(\mathrm{h}-\mathrm{T}_{0} \mathrm{~s}\right)-\left(\mathrm{h}_{0}-\mathrm{T}_{0} \mathrm{~s}_{0}\right)+\frac{\mathrm{C}^{2}}{2}+\mathrm{g}_{\mathrm{G}} \mathrm{Z}_{0}
\end{aligned}
$$

$\mathrm{O}$ balanço de exergia global para um sistema operando em estado estacionário pode ser descrito pela Eq. $\mathrm{H}$ e, em função das considerações adotadas, a Eq. $\mathrm{H}$ pode ser reescrita como a Eq. I:

$$
\begin{aligned}
& \mathrm{E}^{\mathrm{in}}-\mathrm{E}^{\mathrm{ex}}-\mathrm{E}^{\mathrm{Q}}-\mathrm{E}^{\mathrm{L}}=\mathrm{E}^{\mathrm{s}} \\
& \sum \mathrm{m}_{\text {entra }} \cdot \varepsilon_{\text {entra }}^{\mathrm{PH}}=\sum \mathrm{m}_{\text {sai }} \cdot \varepsilon_{\text {sai }}^{\mathrm{PH}}+\sum \mathrm{E}^{\mathrm{Q}}+\mathrm{E}^{\mathrm{L}}
\end{aligned}
$$

A exergia física específica e a exergia relacionada à transferência de calor foram calculadas pelas Eqs. J e K, respectivamente:

$$
\begin{aligned}
\varepsilon^{\mathrm{PH}} & =\left(\mathrm{h}-\mathrm{h}_{0}\right)-\mathrm{T}_{0}\left(\mathrm{~s}-\mathrm{s}_{0}\right) \\
\mathrm{E}^{\mathrm{Q}} & =\mathrm{Q}^{\mathrm{L}} \cdot\left(1-\mathrm{T}_{0} / \mathrm{T}_{\text {sur }}\right)
\end{aligned}
$$

A perda de exergia por irreversibilidades foi calculada pela Eq. L e a exergia relacionada às reações químicas foi calculada pela Eq. M:

$$
\begin{aligned}
& \mathrm{E}^{\mathrm{L}}=\mathrm{T}_{0} \cdot \mathrm{S}_{\text {gen }} \\
& \varepsilon=\mathrm{g}_{\text {reagentes }}^{\mathrm{o}}-\mathrm{g}_{\text {produtos }}^{\mathrm{O}}
\end{aligned}
$$

A eficiência exergética do processo foi calculada conforme a Eq. $\mathrm{N}[10,12]$ :

$$
\eta_{\mathrm{ex}}=\frac{\left|\sum \mathrm{m}_{\text {entra }} \cdot \varepsilon_{\text {entra }}\right|}{\left|\sum \mathrm{m}_{\text {sai }} \cdot \varepsilon_{\text {sai }}\right|}
$$

Considerações adicionais: a primeira alteração na metodologia empregada neste estudo em relação ao trabalho anterior [12] foi a consideração de que não entra $\mathrm{CO}_{2} \mathrm{com}$ a farinha no forno de clínquer. Uma vez que esse gás é gerado anteriormente, especialmente no processo pela queima de combustível em um pré-calcinador, o mesmo não é alimentado ao forno rotativo. $\mathrm{O}$ destino desse gás é sair pela torre de ciclones ao invés de alimentar o forno rotativo. Para realizar essa modificação, considerou-se nula a entrada de $\mathrm{CO}_{2}$ na farinha e o valor da vazão mássica prévia para ele apresentada na Tabela AI foi subtraída na vazão de $\mathrm{CO}_{2}$ dos gases de chaminé. A segunda alteração realizada foi em relação à porcentagem de calcinação que ocorre na etapa de pré-aquecimento. $\mathrm{O}$ trabalho anterior [12] não apresenta o valor da vazão mássica de carbonato de cálcio que entra no forno, apenas a vazão de óxido de cálcio disponível. Isso sugere que foi feita a consideração de $100 \%$ de calcinação do $\mathrm{CaCO}_{3}$ antes mesmo da farinha entrar no forno. Contudo, conforme reportado por profissionais da indústria de cimento, é frequente a realização do processo de clinquerização com $60 \%$ da calcinação ocorrendo antes da farinha entrar no forno. Nesse caso, apenas o restante (40\%) da reação ocorre no forno. Essa modificação no balanço de massa foi realizada respeitando a estequiometria da reação de calcinação $[4,7$, $8,12]$ apresentada na Eq. O, e com base na vazão de $\mathrm{CaO}$ apresentada na Tabela AI (Apêndice):

$$
\mathrm{CaCO}_{3} \rightarrow \mathrm{CaO}+\mathrm{CO}_{2}
$$

O balanço de massa com os valores de vazão mássica para cada corrente nas novas condições está apresentado na Tabela AII (Apêndice). Ainda nesta segunda alteração, as energias e exergias envolvidas na calcinação e nas reações de formação do clínquer (Eqs. P a S) foram acrescentadas ao balanço de energia $[4,7,8,12]$. Os valores referentes às reações de combustão (Eqs. T a W) foram contabilizados como entradas no sistema, enquanto as demais reações foram contabilizadas como saída, seguindo a modelagem realizada em [10]:

$$
\begin{aligned}
& 2 \mathrm{CaO}+\mathrm{SiO}_{2} \rightarrow(\mathrm{CaO})_{2} \mathrm{SiO}_{2} \\
& \mathrm{CaOAl}_{2} \mathrm{O}_{3}+2 \mathrm{CaO} \rightarrow(\mathrm{CaO})_{3} \mathrm{Al}_{2} \mathrm{O}_{3} \\
& \mathrm{CaOAl}_{2} \mathrm{O}_{3}+3 \mathrm{CaO}+\mathrm{Fe}_{2} \mathrm{O}_{3} \rightarrow(\mathrm{CaO})_{4} \mathrm{Al}_{2} \mathrm{O}_{3} \mathrm{Fe}_{2} \mathrm{O} 3 \\
& (\mathrm{CaO})_{2} \mathrm{SiO}_{2}+\mathrm{CaO} \rightarrow(\mathrm{CaO})_{3} \mathrm{SiO}_{2} \\
& \mathrm{C}+\mathrm{O}_{2} \rightarrow \mathrm{CO}_{2} \\
& \mathrm{C}+\frac{1}{2} \mathrm{O}_{2} \rightarrow \mathrm{CO}_{2} \\
& \mathrm{H}_{2}+\frac{1}{2} \mathrm{O}_{2} \rightarrow \mathrm{H}_{2} \mathrm{O} \\
& \mathrm{S}+\mathrm{O}_{2} \rightarrow \mathrm{SO}_{2}
\end{aligned}
$$

Finalmente, a última alteração realizada foi avaliar os balanços de energia e de exergia em função da porcentagem de pré-calcinação que ocorre na farinha antes desta entrar no forno, variando de 0 a $100 \%$. Esses balanços foram realizados considerando a primeira alteração, referente ao $\mathrm{CO}_{2}$. 


\section{RESULTADOS E DISCUSSÃO}

A Tabela AIII (Apêndice) apresenta uma comparação detalhada dos valores encontrados na literatura [12] para o balanço de energia realizado e os que foram recalculados neste trabalho. O desvio percentual e os valores das correntes, considerando apenas os fluxos globais, são apresentados na Tabela I. Foi possível observar que o desvio entre os valores simulados e os valores apresentados na literatura foram inferiores a $10 \%$. Isso indicou que os valores de capacidade calorífica utilizados na simulação foram válidos

Tabela I - Comparação entre os resultados obtidos em [12] e neste trabalho ${ }^{\#}$.

[Table I - Comparison of the results obtained in [12] and the results obtained in this study".]

\begin{tabular}{cccc}
\hline Entrada & $\dot{\mathrm{E}}(\mathrm{kJ} / \mathrm{h})[12]$ & $\dot{\mathrm{E}}(\mathrm{kJ} / \mathrm{h})^{\sharp}$ & Desvio \\
\hline Farinha $(1065 \mathrm{~K})$ & -1301497870 & -1301862985 & $0,03 \%$ \\
Carvão $(318 \mathrm{~K})$ & -41247955 & -45047296 & $9,21 \%$ \\
$\operatorname{Ar}(1373 \mathrm{~K})$ & 272530825 & 289685543 & $6,29 \%$ \\
$\begin{array}{c}\text { Reações de } \\
\text { combustão } \\
\text { Total }\end{array}$ & -245744933 & -243406917 & $0,95 \%$ \\
\hline Saída & -1315959933 & -1300631655 & $1,16 \%$ \\
\hline Elínquer $(1423 \mathrm{~K})$ & -1082377957 & -1030733134 & $4,77 \%$ \\
Poeira $(1320 \mathrm{~K})$ & -86899683 & -87004294 & $0,12 \%$ \\
Gases de chaminé & -110832522 & -117200017 & $5,75 \%$ \\
$(1373 \mathrm{~K})$ & & Simulação & Desvio \\
Total & -1280110161 & -1234937445 & $3,53 \%$ \\
\hline
\end{tabular}

Tabela II - Comparação entre os valores calculados pelas simulações 1 e 2 para o balanço de energia.

[Table II - Comparison between the values calculated by simulations 1 and 2 for the energy balance.]

\begin{tabular}{|c|c|c|}
\hline Entrada & $\begin{array}{l}\text { Simulação } 1 \\
\dot{E}(\mathrm{~kJ} / \mathrm{h})\end{array}$ & $\begin{array}{c}\text { Simulação } 2 \\
\dot{E}(\mathrm{~kJ} / \mathrm{h})\end{array}$ \\
\hline Farinha $(1065 \mathrm{~K})$ & -1301862985 & -1402517475 \\
\hline Carvão (318 K) & $-45047296,38$ & $-45047296,38$ \\
\hline $\operatorname{Ar}(1373 \mathrm{~K})$ & 289685543,1 & 289685543,1 \\
\hline Reações de combustão & -243406917 & -238996651 \\
\hline Total & -1300631655 & -1396875879 \\
\hline Saída & $\begin{array}{c}\text { Simulação } 1 \\
\text { E (kJ/h) }\end{array}$ & $\begin{array}{c}\text { Simulação } 2 \\
\dot{E}(\mathrm{~kJ} / \mathrm{h})\end{array}$ \\
\hline Clínquer (1423 K) & -1030733134 & -1030733134 \\
\hline Poeira $(1320 \mathrm{~K})$ & $-87004293,79$ & $-87004293,79$ \\
\hline $\begin{array}{l}\text { Gases de chaminé (1373 } \\
\text { K) }\end{array}$ & $-117200017,3$ & $-126481964,2$ \\
\hline Reações de clinquerização & - & -1244219392 \\
\hline Calcinação & - & 87694684 \\
\hline Total & -1234937445 & -1196387109 \\
\hline
\end{tabular}

para o sistema estudado. Em um segundo momento foram consideradas as modificações descritas anteriormente. Na Tabela AIV (Apêndice) é apresentada uma comparação detalhada entre os valores simulados já mostrados na Tabela AI (simulação 1) e os valores calculados considerando a primeira e a segunda alteração na metodologia (simulação 2). Na Tabela II a mesma comparação é realizada considerando apenas os valores globais de cada corrente.

A partir dos valores da Tabela AIV e da Eq. E foi possível determinar a eficiência energética de cada condição simulada. Os valores estão expostos na Tabela III. A eficiência energética calculada considerando as reações de calcinação e de clinquerização foi inferior à obtida anteriormente. Isso indicou queéimportante considerá-las no cálculo de eficiência, uma vez que uma parcela considerável da energia fornecida ao sistema é utilizada para que essas reações ocorram. Nesse caso, a redução da eficiência ocorre principalmente em função da reação de calcinação que ocorre dentro do forno, visto que essa é uma reação endotérmica. As reações de clinquerização são exotérmicas, o que contribui para um aumento do valor de eficiência energética. Contudo, são menos impactantes, de forma que ao somar a contribuição das reações o efeito observado foi a redução na eficiência em função da calcinação. Em vista dessa variação na eficiência, infere-se que a não consideração dessas reações no balanço de energia pode omitir a real distribuição desta grandeza no processo. Na Tabela III é possível visualizar os valores numéricos das eficiências energéticas das simulações realizadas neste estudo e os valores reportados em outros trabalhos. A eficiência que apresentou menor desvio $(2,11 \%)$ em relação à simulação 1 foi o trabalho que possui uma configuração similar ao sistema abordado neste estudo [12]. A eficiência energética é um parâmetro muito utilizado por diferentes autores. Porém, não se trata de um parâmetro de fácil comparação quando diferentes sistemas ou equipamentos são analisados. Isto porque existem diferentes maneiras de equacionar esta propriedade e qualquer alteração na configuração do sistema exerce significativa influência no valor encontrado. Assim, acredita-se que os maiores desvios apresentados na Tabela III se explicam em virtude das significativas diferenças entre o sistema analisado neste estudo e as configurações dos demais trabalhos $[10,11,14]$.

Análise exergética: a Tabela AV (Apêndice) apresenta os valores detalhados encontrados na literatura [12] para o balanço de exergia realizado e os que foram recalculados

Tabela III - Valor de eficiência energética.

[Table III - Energy efficiency value.]

\begin{tabular}{ccc}
\hline Referência & Eficiência energética & Desvio \\
\hline Simulação 1 & 0,95 & - \\
Simulação 2 & 0,86 & $9,47 \%$ \\
{$[12]$} & 0,97 & $2,11 \%$ \\
{$[9]$} & 0,558 & $41,26 \%$ \\
{$[11]$} & 0,46 & $51,58 \%$ \\
{$[14]$} & 0,61 & $35,79 \%$ \\
\hline
\end{tabular}


neste trabalho por simulação, bem como o desvio percentual entre eles. Na Tabela IV a mesma comparação é realizada considerando apenas os valores globais de cada corrente. Os desvios encontrados para os valores recalculados no balanço de exergia foram superiores aos observados para o balanço de energia, embora tenham sido usados os mesmos valores de capacidade calorífica no cálculo. O desvio foi significativo especialmente para a exergia que entra no sistema devido ao fluxo de massa de entrada dos componentes do combustível a $318 \mathrm{~K}$. Contudo, essa exergia apresenta valor insignificante em relação à exergia total de entrada no sistema. Isso pode ser confirmado pela análise do desvio percentual para a exergia total de entrada, inferior a $5 \%$.

Tabela IV - Comparação do balanço de exergia realizado em [12] e neste trabalho".

[Table IV - Comparison of the exergy balance made in [12] and obtained in this work ${ }^{\#}$.]

\begin{tabular}{|c|c|c|c|}
\hline Entrada & $\dot{\mathrm{Ex}}(\mathrm{kJ} / \mathrm{h})[12]$ & $\dot{\mathrm{Ex}}(\mathrm{kJ} / \mathrm{h})^{\#}$ & Desvio \\
\hline Farinha $(1065 \mathrm{~K})$ & 47567977,2 & 46605877,09 & $2,02 \%$ \\
\hline Carvão (318 K) & 46865,71 & 14741,92 & $68,54 \%$ \\
\hline $\operatorname{Ar}(1373 \mathrm{~K})$ & 160346734 & 170043633,4 & $6,05 \%$ \\
\hline $\begin{array}{l}\text { Reações de } \\
\text { combustão }\end{array}$ & 234897846,5 & 240913372,9 & $2,56 \%$ \\
\hline Total & 442859423,4 & 457577625,3 & $3,32 \%$ \\
\hline Saída & $\dot{\mathrm{Ex}}(\mathrm{kJ} / \mathrm{h})[12]$ & $\dot{\mathrm{Ex}}(\mathrm{kJ} / \mathrm{h})^{\#}$ & Desvio \\
\hline Clínquer $(1423 \mathrm{~K})$ & 83265566,08 & 61560066,01 & $26,07 \%$ \\
\hline Poeira $(1320 \mathrm{~K})$ & 4846096,33 & 4787012,47 & $1,22 \%$ \\
\hline $\begin{array}{c}\text { Gases de chaminé } \\
(1373 \mathrm{~K})\end{array}$ & 197162483,9 & 194935302,2 & $1,13 \%$ \\
\hline Exergia perdida & 157585277,1 & 196295244,6 & $24,56 \%$ \\
\hline Total & 442859423,4 & 457577625,3 & $3,32 \%$ \\
\hline
\end{tabular}

Assim como na análise energética, o balanço de exergia é analisado para as mesmas condições de simulação 1 e 2 . Os valores calculados para cada simulação estão apresentados nas Tabelas V e AVI (Apêndice). A partir dos valores da Tabela $\mathrm{V}$ e da Eq. $\mathrm{N}$ foi possível determinar a eficiência exergética de cada condição simulada. Os valores estão expostos na Tabela VI. É possível observar que, assim como na análise energética, ocorreu uma redução no cálculo da eficiência. $\mathrm{O}$ mesmo raciocínio é válido, visto que novamente o impacto da reação de calcinação é significativo no valor da eficiência. Na Tabela VI é possível visualizar os valores numéricos das eficiências exergéticas das simulações realizadas neste estudo e em outros trabalhos. A eficiência exergética que apresentou menor desvio $(12,98 \%)$ em relação à simulação 1 foi o trabalho que possui a mesma configuração do sistema deste estudo. As eficiências exergéticas (Tabela VI) apresentaram valores inferiores, quando comparados aos valores das eficiências energéticas (Tabela III). Tal resultado se explica na efetividade da análise exergética em evidenciar perdas de energia (irreversibilidades) as quais o balanço de energia é incapaz de detectar.
Tabela V - Exergia de entrada e saída para as Simulações 1 e 2. [Table V - Inlet and outlet exergy for simulations 1 and 2.]

\begin{tabular}{|c|c|c|}
\hline Entrada & 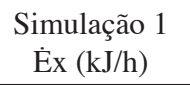 & 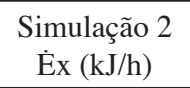 \\
\hline Farinha (1065 K) & 46605877,09 & 45405240,59 \\
\hline Carvão (318 K) & 14741,92 & 14741,92 \\
\hline $\operatorname{Ar}(1373 \mathrm{~K})$ & 170043633,4 & 170043633,4 \\
\hline $\begin{array}{l}\text { Reações de } \\
\text { combustão }\end{array}$ & 240913372,9 & 238046913 \\
\hline Total & 457577625,3 & 453510528,8 \\
\hline Saída & $\begin{array}{l}\text { Simulação } 1 \\
\dot{\mathrm{E}}(\mathrm{kJ} / \mathrm{h})\end{array}$ & $\begin{array}{l}\text { Simulação } 2 \\
\dot{\mathrm{E}}(\mathrm{kJ} / \mathrm{h})\end{array}$ \\
\hline Clínquer (1423 K) & 61560066,01 & 61560066,01 \\
\hline Poeira $(1320 \mathrm{~K})$ & 4787012,47 & 4787012,47 \\
\hline $\begin{array}{l}\text { Gases de chaminé } \\
\qquad(1373 \mathrm{~K})\end{array}$ & 194935302,2 & 195759852,4 \\
\hline $\begin{array}{c}\text { Reações de } \\
\text { clinquerização }\end{array}$ & - & 262106930,9 \\
\hline Calcinação & - & $-64380006,54$ \\
\hline Exergia perdida & 196295244,6 & 229810625,7 \\
\hline Total & 457577625,3 & 453510528,8 \\
\hline
\end{tabular}

Tabela VI - Valor de eficiência exergética. [Table VI - Exergetic efficiency value.]

\begin{tabular}{ccc}
\hline Referência & Eficiência exergética & Desvio \\
\hline Simulação 1 & 0,57 & - \\
Simulação 2 & 0,49 & $14,03 \%$ \\
[12] & 0,644 & $12,98 \%$ \\
[9] & 0,387 & $32,10 \%$ \\
{$[11]$} & 0,35 & $38,59 \%$ \\
{$[14]$} & 0,49 & $14,03 \%$ \\
\hline
\end{tabular}

Influência da reação de calcinação nas eficiências energética e exergética: os resultados obtidos neste trabalho apontam que o maior impacto nos cálculos das eficiências energética e exergética ocorreu devido à adição da reação de calcinação nos balanços. Em vista disso, infere-se que é relevante analisar como essas eficiências são afetadas em função da porcentagem dessa reação que ocorre no forno. Na Fig. 3 é apresentado o comportamento do valor de cada eficiência em função da porcentagem da reação de descarbonatação do calcário que ocorreu antes que a farinha entrasse no forno. Ou seja, para $0 \%$, há presença de apenas carbonato de cálcio na farinha e ausência de óxido de cálcio. Para 100\%, toda a calcinação já aconteceu, de forma que na farinha que entra no forno há apenas óxido de cálcio. Conforme esperado, quanto maior é a porcentagem da reação que ocorre antes do forno, maior é a sua eficiência energética, visto que menor fração da energia fornecida ao equipamento é utilizada para a realização dessa reação endotérmica. Esse é um dos 


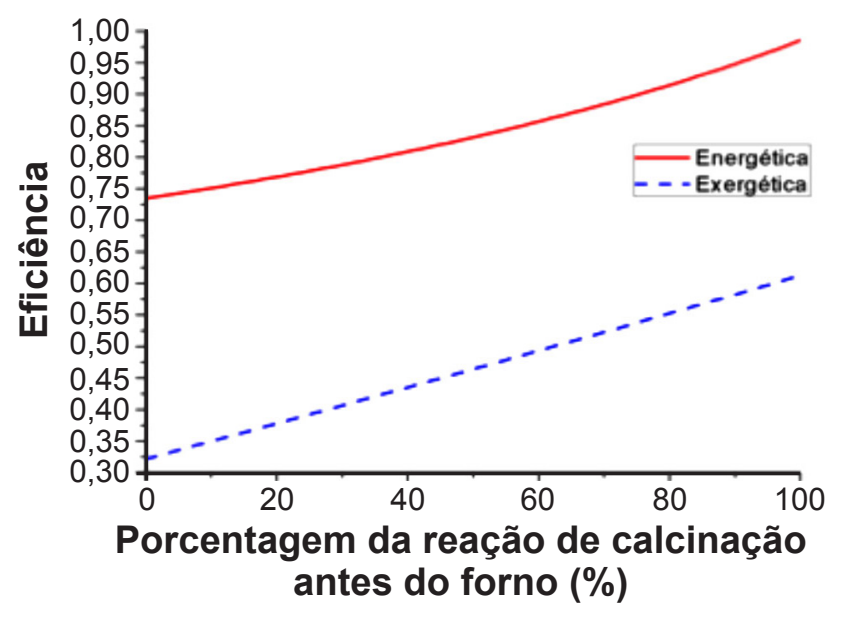

Figura 3: Eficiência energética e exergética do forno em função da porcentagem da reação de calcinação que ocorreu antes do forno rotativo.

[Figure 3: Energy and exergetic efficiency as a function of the percentage of the calcination reaction that occurred before the kiln.]

motivos pelo qual a indústria de cimento busca realizar grande parte da calcinação na etapa de pré-aquecimento. Tal ação traz benefícios como: possibilidade de aumento da capacidade do forno; redução da carga térmica nos refratários e maior estabilidade do forno [20]. Assim como na eficiência energética, a eficiência exergética também se comportou conforme esperado. A ocorrência da calcinação dentro do forno resultou em uma redução da eficiência exergética. Isso porque parte da energia útil fornecida ao forno, que poderia ser utilizada para realização de trabalho, é gasta para a realização da reação de decomposição do carbonato de cálcio.

\section{CONCLUSÕES}

A metodologia e os valores de capacidade calorífica utilizados se mostraram adequados para a análise exergética e energética da realidade industrial avaliada, visto que os valores calculados por simulação apresentaram pequeno desvio em relação aos fornecidos pela literatura. Foi possível concluir que as reações de formação do clínquer e a reação de calcinação apresentam influência significativa nos balanços de energia e exergia. Especificamente em relação à calcinação, constatouse que é desejado que ela ocorra na menor quantidade possível dentro do forno. Essa quantidade deve ser avaliada para cada realidade industrial, visto que não são apenas fatores energéticos que influenciam na operação deste equipamento.

\section{AGRADECIMENTOS}

Os autores agradecem o apoio financeiro da CAPES e a FAPEMIG por meio do Edital FAPEMIG 01/2016 Demanda Universal, Processo TEC APQ 00914-16.

\section{LISTA DE VARIÁVEIS}

$\begin{array}{ll}\mathrm{C} & \text { Velocidade de fluxo } \\ \dot{\mathrm{E}} & \text { Taxa de energia } \\ \mathrm{E}^{\text {ex }} & \text { Exergia de saída } \\ \mathrm{E}^{\text {in }} & \text { Exergia de entrada } \\ \mathrm{E}^{\mathrm{L}} & \text { Perda de exergia por irreversibilidades } \\ \mathrm{E}^{\mathrm{Q}} & \text { Exergia de transferência de calor } \\ \mathrm{E}^{\mathrm{S}} & \text { Exergia do sistema } \\ \dot{\mathrm{Ex}}_{\mathrm{x}}^{\mathrm{KN}} & \text { Taxa de exergia } \\ \varepsilon_{\mathrm{f}}^{\mathrm{KN}} & \text { Exergia específica cinética } \\ \varepsilon_{\mathrm{f}}^{\mathrm{PH}} & \text { Exergia específica física }\end{array}$

$\varepsilon_{\text {sai }}^{\mathrm{PH}} \quad$ Exergia física específica de cada componente que entra no volume de controle

$\varepsilon_{\text {entra }}^{\mathrm{PH}} \quad$ Exergia física específica de cada componente que deixa o volume de controle

$\varepsilon_{\mathrm{f}}^{\text {PT }} \quad$ Exergia específica potencial

$\varepsilon \quad$ Exergia

$\mathrm{g}_{\text {reagentes }}^{\circ}$ Energia livre de Gibbs específica de formação dos reagentes

$\mathrm{g}_{\text {produtos }}^{\circ}$ Energia livre de Gibbs específica de formação dos produtos

$\mathrm{g}_{\mathrm{G}} \quad$ Aceleração da gravidade

h Entalpia específica

$\mathrm{h}_{0} \quad$ Entalpia específica ambiente

$\mathrm{h}_{\text {entra }} \quad$ Entalpia específica de cada componente que entra no no volume de controle

$\mathrm{h}_{\text {sai }} \quad$ Entalpia específica de cada componente que deixa o volume de controle

$\mathrm{m}_{\text {entra }} \quad$ Vazão mássica de cada componente que entra no volume de controle

$\mathrm{m}_{\text {sai }} \quad$ Vazão mássica de cada componente que deixa o volume de controle

$\mathrm{m}_{\text {farinha }}$ Vazão mássica de cada componente da farinha

$\mathrm{m}_{\text {combustível }}$ Vazão mássica de cada componente do combustível

$\mathrm{m}_{\mathrm{ar}} \quad$ Vazão mássica de cada componente do ar

$\mathrm{m}_{\text {clínquer }} \quad$ Vazão mássica de cada componente do clínquer

$\mathrm{m}_{\text {poira }} \quad$ Vazão mássica de cada componente da poeira

$\mathrm{m}_{\text {gases }} \quad$ Vazão mássica de cada componente dos gases

$\eta_{\mathrm{e}} \quad$ Eficiência energética

$\eta_{\text {ex }} \quad$ Eficiência exergética

Q Calor que deixa o volume de controle por condução, convecção e irradiação

$\mathrm{Q}_{\mathrm{L}} \quad$ Perda de calor pelas paredes do forno rotativo

s $\quad$ Entropia específica 


$\begin{array}{ll}\mathrm{s}_{0} & \text { Entropia específica ambiente } \\ \mathrm{S}_{\text {gen }} & \text { Termo de geração de entropia } \\ \mu_{\mathrm{i} 0} & \text { Potencial químico da espécie i ambiente } \\ \mu_{\mathrm{i} 00} & \text { Potencial químico da espécie i no equilíbrio } \\ \mathrm{Z}_{0} & \text { Altura }\end{array}$

\section{APÊNDICE}

Em todas as tabelas contidas neste Apêndice é considerada a seguinte nomenclatura: $\mathrm{C}_{3} \mathrm{~S}-3 \mathrm{CaO} . \mathrm{SiO}_{2} ; \mathrm{C}_{2} \mathrm{~S}$ - $2 \mathrm{CaO} \cdot \mathrm{SiO}_{2} ; \mathrm{C}_{3} \mathrm{~A}-3 \mathrm{CaO} \cdot \mathrm{Al}_{2} \mathrm{O}_{3} ; \mathrm{C}_{4} \mathrm{AF}-4 \mathrm{CaO} \cdot \mathrm{Al}_{2} \mathrm{O}_{3} \cdot \mathrm{Fe}_{2} \mathrm{O}_{3}$.

Tabela AI - Balanço de massa para o forno rotativo por componente; adaptado de [12].

[Table AI - Rotary kiln mass balance by component; adapted from [12].]

\begin{tabular}{|c|c|c|c|}
\hline \multicolumn{2}{|c|}{ Vazão de entrada $(\mathrm{kg} / \mathrm{h})$} & \multicolumn{2}{|c|}{ Vazão de saída $(\mathrm{kg} / \mathrm{h})$} \\
\hline \multicolumn{2}{|c|}{ Farinha $(1065 \mathrm{~K})$} & \multicolumn{2}{|c|}{ Clínquer (1423 K) } \\
\hline $\mathrm{CaO}$ & 68948,80 & $\mathrm{C}_{3} \mathrm{~S}$ & 57111,30 \\
\hline $\mathrm{CO}_{2}$ & 20467,70 & $\mathrm{C}_{2} \mathrm{~S}$ & 13438,50 \\
\hline $\mathrm{SiO}_{2}$ & 18924,80 & $\mathrm{C}_{3} \mathrm{~A}$ & 9104,70 \\
\hline $\mathrm{Al}_{2} \mathrm{O}_{3}$ & 5520,70 & $\mathrm{C}_{4} \mathrm{AF}$ & 10955,40 \\
\hline $\mathrm{Fe}_{2} \mathrm{O}_{3}$ & 3676,50 & $\mathrm{MgO}$ & 1357,80 \\
\hline $\mathrm{MgO}$ & 1735,80 & $\mathrm{SO}_{3}$ & 232,50 \\
\hline $\mathrm{K}_{2} \mathrm{O}$ & 843,80 & $\mathrm{~K}_{2} \mathrm{O}$ & 660,30 \\
\hline $\mathrm{SO}_{3}$ & 241,10 & $\mathrm{Na}_{2} \mathrm{O}$ & 139,50 \\
\hline $\mathrm{Na}_{2} \mathrm{O}$ & 180,80 & Total & 93000,00 \\
\hline Total & 120540,00 & \multicolumn{2}{|c|}{ Poeira $(1320 \mathrm{~K})$} \\
\hline \multicolumn{2}{|c|}{ Carvão (318 K) } & $\mathrm{CaO}$ & 7427,79 \\
\hline $\mathrm{C}$ & 5668,25 & $\mathrm{SiO}_{2}$ & 40,30 \\
\hline$S$ & 389,50 & $\mathrm{Al}_{2} \mathrm{O}_{3}$ & 83,75 \\
\hline $\mathrm{N}_{2}$ & 164,00 & $\mathrm{Fe}_{2} \mathrm{O}_{3}$ & 291,79 \\
\hline $\mathrm{H}_{2}$ & 451,00 & $\mathrm{MgO}$ & 410,80 \\
\hline $\mathrm{O}_{2}$ & 461,25 & $\mathrm{SO}_{3}$ & 78,30 \\
\hline $\mathrm{H}_{2} \mathrm{O}$ & 1610,27 & $\mathrm{~K}_{2} \mathrm{O}$ & 204,00 \\
\hline $\mathrm{CaO}$ & 38,95 & $\mathrm{Na}_{2} \mathrm{O}$ & 41,30 \\
\hline $\mathrm{SiO}_{2}$ & 831,28 & Total & 8578,03 \\
\hline $\mathrm{Al}_{2} \mathrm{O}_{3}$ & 297,25 & \multicolumn{2}{|c|}{ Gases de chaminé ( $1373 \mathrm{~K}$ ) } \\
\hline $\mathrm{Fe}_{2} \mathrm{O}_{3}$ & 215,25 & $\mathrm{CO}_{2}$ & 40693,43 \\
\hline $\mathrm{MgO}$ & 32,80 & $\mathrm{CO}$ & 355,00 \\
\hline $\mathrm{K}_{2} \mathrm{O}$ & 20,50 & $\mathrm{SO}_{2}$ & 779,00 \\
\hline $\mathrm{SO}_{3}$ & 69,70 & $\mathrm{H}_{2} \mathrm{O}$ & 5669,30 \\
\hline Total & 10250,00 & $\mathrm{O}_{2}$ & 38206,02 \\
\hline \multicolumn{2}{|c|}{$\operatorname{Ar}(1373$ e $298 \mathrm{~K})$} & $\mathrm{N}_{2}$ & 186558,22 \\
\hline $\mathrm{O}_{2}$ & 56654,78 & Total & 272260,97 \\
\hline $\mathrm{N}_{2}$ & 186394,22 & & \\
\hline Total & 243049,00 & & \\
\hline Somatório & 373839,00 & Somatório & 373839,00 \\
\hline
\end{tabular}

Tabela AII - Balanço de massa modificado para o forno rotativo por componente.

[Table AII - Modified rotary kiln mass balance by component.]

\begin{tabular}{|c|c|c|c|}
\hline \multicolumn{2}{|c|}{ Vazão de entrada $(\mathrm{kg} / \mathrm{h})$} & \multicolumn{2}{|c|}{ Vazão de saída $(\mathrm{kg} / \mathrm{h})$} \\
\hline \multicolumn{2}{|c|}{ Farinha $(1065 \mathrm{~K})$} & \multicolumn{2}{|c|}{ Clínquer (1423 K) } \\
\hline $\mathrm{CaCO}_{3}$ & 49223,91 & $\mathrm{C}_{3} \mathrm{~S}$ & 57111,30 \\
\hline $\mathrm{CaO}$ & 41396,28 & $\mathrm{C}_{2} \mathrm{~S}$ & 13438,50 \\
\hline $\mathrm{CO}_{2}$ & 0 & $\mathrm{C}_{3} \mathrm{~A}$ & 9104,70 \\
\hline $\mathrm{SiO}_{2}$ & 18924,80 & $\mathrm{C}_{4} \mathrm{AF}$ & 10955,40 \\
\hline $\mathrm{Al}_{2} \mathrm{O}_{3}$ & 5520,70 & $\mathrm{MgO}$ & 1357,80 \\
\hline $\mathrm{Fe}_{2} \mathrm{O}_{3}$ & 3676,50 & $\mathrm{SO}_{3}$ & 232,50 \\
\hline $\mathrm{MgO}$ & 1735,80 & $\mathrm{~K}_{2} \mathrm{O}$ & 660,30 \\
\hline $\mathrm{K}_{2} \mathrm{O}$ & 843,80 & $\mathrm{Na}_{2} \mathrm{O}$ & 139,50 \\
\hline $\mathrm{SO}_{3}$ & 241,10 & Total & 93000,00 \\
\hline $\mathrm{Na}_{2} \mathrm{O}$ & 180,80 & \multicolumn{2}{|c|}{ Poeira $(1320 \mathrm{~K})$} \\
\hline Total & 121716,69 & $\mathrm{CaO}$ & 7427,79 \\
\hline \multicolumn{2}{|c|}{ Carvão (318 K) } & $\mathrm{SiO}_{2}$ & 40,30 \\
\hline $\mathrm{C}$ & 5668,25 & $\mathrm{Al}_{2} \mathrm{O}_{3}$ & 83,75 \\
\hline$S$ & 389,50 & $\mathrm{Fe}_{2} \mathrm{O}_{3}$ & 291,79 \\
\hline $\mathrm{N}_{2}$ & 164,00 & $\mathrm{MgO}$ & 410,80 \\
\hline $\mathrm{H}_{2}$ & 451,00 & $\mathrm{SO}_{3}$ & 78,30 \\
\hline $\mathrm{O}_{2}$ & 461,25 & $\mathrm{~K}_{2} \mathrm{O}$ & 204,00 \\
\hline $\mathrm{H}_{2} \mathrm{O}$ & 1610,27 & $\mathrm{Na}_{2} \mathrm{O}$ & 41,30 \\
\hline $\mathrm{CaO}$ & 38,95 & Total & 8578,03 \\
\hline $\mathrm{SiO}_{2}$ & 831,28 & \multicolumn{2}{|c|}{ Gases de chaminé (1373 K) } \\
\hline $\mathrm{Al}_{2} \mathrm{O}_{3}$ & 297,25 & $\mathrm{CO}_{2}$ & 41870.12 \\
\hline $\mathrm{Fe}_{2} \mathrm{O}_{3}$ & 215,25 & $\mathrm{CO}$ & 355,00 \\
\hline $\mathrm{MgO}$ & 32,80 & $\mathrm{SO}_{2}$ & 779,00 \\
\hline $\mathrm{K}_{2} \mathrm{O}$ & 20,50 & $\mathrm{H}_{2} \mathrm{O}$ & 5669,30 \\
\hline $\mathrm{SO}_{3}$ & 69,70 & $\mathrm{O}_{2}$ & 38206,02 \\
\hline Total & 10250,00 & $\mathrm{~N}_{2}$ & 186558,22 \\
\hline \multicolumn{2}{|c|}{$\operatorname{Ar}(1373$ e $298 \mathrm{~K})$} & Total & 273442,02 \\
\hline $\mathrm{O}_{2}$ & 56654,78 & & \\
\hline $\mathrm{N}_{2}$ & 186394,22 & & \\
\hline Total & 243049,00 & & \\
\hline Somatório & 375015,69 & Somatório & 375015,69 \\
\hline
\end{tabular}


Tabela AIII - Comparação detalhada entre os resultados obtidos em [12] e neste trabalho" por componente. [Table AIII - Comparison of the results obtained in [12] and the results obtained in this study" by component.]

\begin{tabular}{|c|c|c|c|c|c|c|c|}
\hline Entrada & $\begin{array}{c}\mathrm{m}_{\mathrm{in}} \mathrm{h}_{\text {in }}(\mathrm{kJ} / \mathrm{h}) \\
{[12]}\end{array}$ & $\mathrm{m}_{\text {in }} \mathrm{h}_{\text {in }}(\mathrm{kJ} / \mathrm{h})^{\#}$ & Erro & Saída & $\begin{array}{c}\mathrm{m}_{\mathrm{ex}} \mathrm{h}_{\mathrm{ex}}(\mathrm{kJ} / \mathrm{h}) \\
{[12]}\end{array}$ & $\begin{array}{c}\text { Simulação } \\
\mathrm{m}_{\mathrm{ex}} \mathrm{h}_{\mathrm{ex}}(\mathrm{kJ} / \mathrm{h})\end{array}$ & Erro \\
\hline \multicolumn{4}{|c|}{ Farinha $(1065 \mathrm{~K})$} & \multicolumn{4}{|c|}{ Clínquer (1423 K) } \\
\hline $\mathrm{CaO}$ & -733180855 & -734136892 & $0,13 \%$ & $\mathrm{C}_{3} \mathrm{~S}$ & -654910126 & $-641770969,4$ & $2,01 \%$ \\
\hline $\mathrm{CO}_{2}$ & -165814978 & -1658262561 & $0,01 \%$ & $\mathrm{C}_{2} \mathrm{~S}$ & $-159973113,5$ & $-155128182,2$ & $3,03 \%$ \\
\hline $\mathrm{SiO}_{2}$ & -271205631 & -270561138 & $0,24 \%$ & $\mathrm{C}_{3} \mathrm{~A}$ & -110598797 & $-110386111,6$ & $0,19 \%$ \\
\hline $\mathrm{Al}_{2} \mathrm{O}_{3}$ & $-86066608,9$ & $-86067166,6$ & $0,00 \%$ & $(\mathrm{CaO})_{4}$ & $-85641719,1$ & $-52028662,87$ & $39,25 \%$ \\
\hline $\mathrm{Fe}_{2} \mathrm{O}_{3}$ & -16449764 & -16435232 & $0,09 \%$ & $\mathrm{Al}_{2} \mathrm{O}_{3}$ & $-34793961,43$ & $-34813884,02$ & $0,06 \%$ \\
\hline $\mathrm{MgO}$ & $-24324806,9$ & $-24327800,5$ & $0,01 \%$ & $\mathrm{Fe}_{2} \mathrm{O}_{3}$ & $-14961104,2$ & $-14957143,63$ & $0,03 \%$ \\
\hline $\mathrm{K}_{2} \mathrm{O}$ & $-2399007,78$ & $-2561475,1$ & $6,77 \%$ & $\mathrm{MgO}$ & $-18399058,99$ & $-18397100,35$ & $0,01 \%$ \\
\hline $\mathrm{SO}_{3}$ & $-1035186,96$ & $-1035123,16$ & $0,01 \%$ & $\mathrm{SO}_{3}$ & $-917059,05$ & $-931653,74$ & $1,59 \%$ \\
\hline $\mathrm{Na}_{2} \mathrm{O}$ & $-1021031,84$ & $-911901,54$ & $10,69 \%$ & $\mathrm{~K}_{2} \mathrm{O}$ & $-1608781,33$ & $-1694811,2$ & $5,35 \%$ \\
\hline \multirow[t]{2}{*}{ Total } & -1301497870 & -1301862985 & $0,03 \%$ & $\mathrm{Na}_{2} \mathrm{O}$ & $-574236,41$ & $-624615,38$ & $8,77 \%$ \\
\hline & \multicolumn{2}{|c|}{ Carvão (318 K) } & & \multicolumn{2}{|r|}{-1082377957} & -1030733134 & $4,77 \%$ \\
\hline $\mathrm{C}$ & 95396,65 & 81153,66 & $14,93 \%$ & \multicolumn{4}{|c|}{ Poeira $(1320 \mathrm{~K})$} \\
\hline S & 5639,96 & 5570,6 & $1,23 \%$ & $\mathrm{CaO}$ & $-77148740,83$ & $-77235542,41$ & $0,11 \%$ \\
\hline $\mathrm{N}_{2}$ & 3409,56 & 3410,71 & $0,03 \%$ & $\mathrm{SiO}_{2}$ & $-563990,44$ & $-563948,52$ & $0,01 \%$ \\
\hline $\mathrm{H}_{2}$ & 129152,87 & 129298,38 & $0,11 \%$ & $\mathrm{Al}_{2} \mathrm{O}_{3}$ & $-1278828,16$ & $-1278782,43$ & $0,00 \%$ \\
\hline $\mathrm{O}_{2}$ & 8570,03 & 8486,07 & $0,98 \%$ & $\mathrm{Fe}_{2} \mathrm{O}_{3}$ & $-1239515,17$ & $-1238497,89$ & $0,08 \%$ \\
\hline $\mathrm{H}_{2} \mathrm{O}$ & $-21561515,3$ & $-25413874,6$ & $17,87 \%$ & $\mathrm{MgO}$ & $-5622233,45$ & $-5621775,11$ & $0,01 \%$ \\
\hline $\mathrm{CaO}$ & $-440481,66$ & $-440527,78$ & $0,01 \%$ & $\mathrm{SO}_{3}$ & $-316804,15$ & $-321721,36$ & $1,55 \%$ \\
\hline $\mathrm{SiO}_{2}$ & $-12589378,2$ & $-12519503,9$ & $0,56 \%$ & $\mathrm{~K}_{2} \mathrm{O}$ & $-520901,76$ & $-552287,42$ & $6,03 \%$ \\
\hline $\mathrm{Al}_{2} \mathrm{O}_{3}$ & $-4880170,24$ & $-4880441,46$ & $0,01 \%$ & $\mathrm{Na}_{2} \mathrm{O}$ & $-208668,66$ & $-191738,65$ & $8,11 \%$ \\
\hline $\mathrm{Fe}_{2} \mathrm{O}_{3}$ & $-1108035,97$ & $-1109874,92$ & $0,17 \%$ & Total & $-86899682,62$ & $-87004293,79$ & $0,12 \%$ \\
\hline $\mathrm{MgO}$ & $-488581,26$ & $-488677,91$ & $0,02 \%$ & & Gases de cham & iné (1373 K) & \\
\hline $\mathrm{K}_{2} \mathrm{O}$ & $-78338,7$ & $-78667,08$ & $0,42 \%$ & $\mathrm{CO}_{2}$ & $-313599035,1$ & -319812250 & $1,98 \%$ \\
\hline $\mathrm{SO}_{3}$ & $-343622,39$ & $-343648,16$ & $0,01 \%$ & $\mathrm{CO}$ & $-964822,55$ & $-1018536,1$ & $5,57 \%$ \\
\hline Total & $-41247954,6$ & $-45047296,4$ & $9,21 \%$ & $\mathrm{SO}_{2}$ & $-2941659,8$ & $-2977060,35$ & $1,20 \%$ \\
\hline & $\operatorname{Ar}(1373$ & & & $\mathrm{H}_{2} \mathrm{O}$ & $-62819585,74$ & $-62804698,06$ & $0,02 \%$ \\
\hline $\mathrm{O}_{2} *$ & 59888721,99 & 62869784,88 & $4,98 \%$ & $\mathrm{O}_{2} * *$ & 42982918,68 & 42397203,88 & $1,36 \%$ \\
\hline $\mathrm{N}_{2}^{*}$ & 212642102,9 & 226815758,2 & $6,67 \%$ & $\mathrm{~N}_{2} * *$ & 226509662,8 & 227015323,3 & $0,22 \%$ \\
\hline Total & 272530824,9 & 289685543,1 & $6,29 \%$ & Total & $-110832521,7$ & $-117200017,3$ & $5,75 \%$ \\
\hline & Energia de re & eação ${ }^{\forall}$ & & & & & \\
\hline $\mathrm{C}\left(\right.$ para $\left.\mathrm{CO}_{2}\right)$ & -185879172 & -185710496 & $0,09 \%$ & & & & \\
\hline $\mathrm{H}_{2}\left(\right.$ para $\left.\mathrm{H}_{2} \mathrm{O}\right)$ & -56247620 & -54100849 & $3,82 \%$ & & & & \\
\hline $\mathrm{S}\left(\right.$ para $\left.\mathrm{SO}_{2}\right)$ & -3618141 & -3595571 & $0,62 \%$ & & & & \\
\hline Total & -245744933 & -243406917 & $0,95 \%$ & & & & \\
\hline Somatório & -1315959933 & -1300631655 & $1,16 \%$ & Somatório & -1280110161 & -1234937445 & $3,53 \%$ \\
\hline & $(-365544 \mathrm{~kW})$ & $(-361287 \mathrm{~kW})$ & & & $(-355586 \mathrm{~kW})$ & $(-343038 \mathrm{~kW})$ & \\
\hline
\end{tabular}


Tabela AIV - Comparação detalhada entre os valores calculados pelas simulações 1 e 2 para o balanço de energia por componente.

[Table AIV - Comparison between the values calculated by simulations 1 and 2 for the energy balance by component.]

\begin{tabular}{|c|c|c|c|c|c|}
\hline Entrada & $\begin{array}{l}\text { Simulação } 1 \\
\mathrm{~m}_{\text {in }} \mathrm{h}_{\text {in }}(\mathrm{kJ} / \mathrm{h})\end{array}$ & $\begin{array}{l}\text { Simulação } 2 \\
\mathrm{~m}_{\text {in }} \mathrm{h}_{\text {in }}(\mathrm{kJ} / \mathrm{h})\end{array}$ & Saída & $\begin{array}{l}\text { Simulação } 1 \\
\mathrm{~m}_{\mathrm{ex}} \mathrm{h}_{\mathrm{ex}}(\mathrm{kJ} / \mathrm{h})\end{array}$ & $\begin{array}{l}\text { Simulação } 2 \\
\mathrm{~m}_{\mathrm{ex}} \mathrm{h}_{\mathrm{ex}}(\mathrm{kJ} / \mathrm{h})\end{array}$ \\
\hline \multicolumn{3}{|c|}{ Farinha $(1065 \mathrm{~K})$} & \multicolumn{3}{|c|}{ Clínquer $(1423 \mathrm{~K})$} \\
\hline $\mathrm{CaO}$ & $-734136891,5$ & $-440482134,9$ & $\mathrm{C}_{3} \mathrm{~S}$ & $-641770969,4$ & $-641770969,4$ \\
\hline $\mathrm{CO}_{2}$ & -1658262561 & - & $\mathrm{C}_{2} \mathrm{~S}$ & $-155128182,2$ & $-155128182,2$ \\
\hline $\mathrm{SiO}_{2}$ & $-270561138,4$ & $-270561138,4$ & $\mathrm{C}_{3} \mathrm{~A}$ & $-110386111,6$ & $-110386111,6$ \\
\hline $\mathrm{Al}_{2} \mathrm{O}_{3}$ & $-86067166,56$ & $-86067166,56$ & $(\mathrm{CaO})_{4}$ & $-52028662,87$ & $-52028662,87$ \\
\hline $\mathrm{Fe}_{2} \mathrm{O}_{3}$ & $-16435232,04$ & $-16435232,04$ & $\mathrm{Al}_{2} \mathrm{O}_{3}$ & $-34813884,02$ & $-34813884,02$ \\
\hline $\mathrm{MgO}$ & $-24327800,47$ & $-24327800,47$ & $\mathrm{Fe}_{2} \mathrm{O}_{3}$ & $-14957143,63$ & $-14957143,63$ \\
\hline $\mathrm{K}_{2} \mathrm{O}$ & $-2561475,1$ & $-2561475,1$ & $\mathrm{MgO}$ & $-18397100,35$ & $-18397100,35$ \\
\hline $\mathrm{SO}_{3}$ & $-1035123,16$ & $-1035123,16$ & $\mathrm{SO}_{3}$ & $-931653,74$ & $-931653,74$ \\
\hline $\mathrm{Na}_{2} \mathrm{O}$ & $-911901,54$ & $-911901,54$ & $\mathrm{~K}_{2} \mathrm{O}$ & $-1694811,2$ & $-1694811,2$ \\
\hline $\mathrm{CaCO}_{3}$ & - & -560135503 & $\mathrm{Na}_{2} \mathrm{O}$ & $-624615,38$ & $-624615,38$ \\
\hline Total & -1301862985 & -1402517475 & Total & -1030733134 & -1030733134 \\
\hline \multicolumn{3}{|c|}{ Carvão (318 K) } & \multicolumn{3}{|c|}{ Poeira $(1320 \mathrm{~K})$} \\
\hline $\mathrm{C}$ & 81153,66 & 81153,66 & $\mathrm{CaO}$ & $-77235542,41$ & $-77235542,41$ \\
\hline$S$ & 5570,6 & 5570,6 & $\mathrm{SiO}_{2}$ & $-563948,52$ & $-563948,52$ \\
\hline $\mathrm{N}_{2}$ & 3410,71 & 3410,71 & $\mathrm{Al}_{2} \mathrm{O}_{3}$ & $-1278782,43$ & $-1278782,43$ \\
\hline $\mathrm{H}_{2}$ & 129298,38 & 129298,38 & $\mathrm{Fe}_{2} \mathrm{O}_{3}$ & $-1238497,89$ & $-1238497,89$ \\
\hline $\mathrm{O}_{2}$ & 8486,07 & 8486,07 & $\mathrm{MgO}$ & $-5621775,11$ & $-5621775,11$ \\
\hline $\mathrm{H}_{2} \mathrm{O}$ & $-25413874,57$ & $-25413874,57$ & $\mathrm{SO}_{3}$ & $-321721,36$ & $-321721,36$ \\
\hline $\mathrm{CaO}$ & $-440527,78$ & $-440527,78$ & $\mathrm{~K}_{2} \mathrm{O}$ & $-552287,42$ & $-552287,42$ \\
\hline $\mathrm{SiO}_{2}$ & $-12519503,91$ & $-12519503,91$ & $\mathrm{Na}_{2} \mathrm{O}$ & $-191738,65$ & $-191738,65$ \\
\hline $\mathrm{Al}_{2} \mathrm{O}_{3}$ & $-4880441,46$ & $-4880441,46$ & Total & $-87004293,79$ & $-87004293,79$ \\
\hline $\mathrm{Fe}_{2} \mathrm{O}_{3}$ & $-1109874,92$ & $-1109874,92$ & \multicolumn{3}{|c|}{ Gases de chaminé (1373 K) } \\
\hline $\mathrm{MgO}$ & $-488677,91$ & $-488677,91$ & $\mathrm{CO}_{2}$ & -319812250 & $-329094196,9$ \\
\hline $\mathrm{K}_{2} \mathrm{O}$ & $-78667,08$ & $-78667,08$ & $\mathrm{CO}$ & $-1018536,1$ & $-1018536,1$ \\
\hline $\mathrm{SO}_{3}$ & $-343648,16$ & $-343648,16$ & $\mathrm{SO}_{2}$ & $-2977060,35$ & $-2977060,35$ \\
\hline \multirow[t]{2}{*}{ Total } & $-45047296,38$ & $-45047296,38$ & $\mathrm{H}_{2} \mathrm{O}$ & $-62804698,06$ & $-62804698,06$ \\
\hline & $\operatorname{Ar}(1373 \mathrm{~K})$ & & $\mathrm{O}_{2}^{* *}$ & 42397203,88 & 42397203,88 \\
\hline $\mathrm{O}_{2}^{*}$ & 62869784,88 & 62869784,88 & $\mathrm{~N}_{2} * *$ & 227015323,3 & 227015323,3 \\
\hline $\mathrm{N}_{2}^{*}$ & 226815758,2 & 226815758,2 & Total & $-117200017,3$ & $-126481964,2$ \\
\hline Total & 289685543,1 & 289685543,1 & \multicolumn{3}{|c|}{ Energia de reação ${ }^{\forall}$} \\
\hline \multicolumn{3}{|c|}{ Energia de reação ${ }^{\forall}$} & Formação $\mathrm{C}_{3} \mathrm{~S}$ & - & -28283434 \\
\hline $\mathrm{C}\left(\right.$ para $\left.\mathrm{CO}_{2}\right)$ & -185710496 & -180696313 & Formação $\mathrm{C}_{2} \mathrm{~S}$ & - & -9866707 \\
\hline $\mathrm{C}($ para CO) & - & -603918 & Formação $\mathrm{C}_{3} \mathrm{~A}$ & - & -455920 \\
\hline $\mathrm{H}_{2}\left(\right.$ para $\left.\mathrm{H}_{2} \mathrm{O}\right)$ & -54100849 & -54100849 & Formação $\mathrm{C}_{4} \mathrm{AF}$ & - & -1290637 \\
\hline $\mathrm{S}\left(\right.$ para $\left.\mathrm{SO}_{2}\right)$ & -3595571 & -3595571 & Calcinação & - & 87694684 \\
\hline Total & -243406917 & -238996651 & Total & - & 47797986 \\
\hline Somatório & -1300631655 & -1396875879 & Somatório & -1234937445 & -1196387109 \\
\hline
\end{tabular}

* - ar primário + ar secundário; $* *$ - ar residual; ${ }^{\forall}$ - valores para $\dot{E}(\mathrm{~kJ} / \mathrm{h})$. 
Tabela AV - Comparação do balanço de exergia realizado em [12] e neste trabalho" por componente.

[Table AV - Comparison of the exergy balance made in [12] and obtained in this work ${ }^{*}$ by component.]

\begin{tabular}{|c|c|c|c|c|c|c|c|}
\hline Entrada & $\begin{array}{c}\dot{\mathrm{E}}^{\mathrm{PH}}(\mathrm{kJ} / \mathrm{h}) \\
{[12]}\end{array}$ & $\dot{\mathrm{E}}^{\mathrm{PH}}(\mathrm{kJ} / \mathrm{h})^{\#}$ & Erro & Saída & $\begin{array}{c}\dot{\mathrm{E}}^{\mathrm{PH}}(\mathrm{kJ} / \mathrm{h}) \\
{[12]}\end{array}$ & $\dot{\mathrm{E}}^{\mathrm{PH}}(\mathrm{kJ} / \mathrm{h})^{\#}$ & Erro \\
\hline \multicolumn{4}{|c|}{ Farinha (1065 K) } & \multicolumn{4}{|c|}{ Clínquer (1423 K) } \\
\hline $\mathrm{CaO}$ & 24813983,63 & 24621797,15 & $0,77 \%$ & $\mathrm{C}_{3} \mathrm{~S}$ & 38982444,96 & 37380411,89 & $4,11 \%$ \\
\hline $\mathrm{CO}_{2}$ & 9123681,95 & 9016235,75 & $1,18 \%$ & $\mathrm{C}_{2} \mathrm{~S}$ & 9460417,06 & 8928845,66 & $5,62 \%$ \\
\hline $\mathrm{SiO}_{2}$ & 8340159,36 & 7865850,11 & $5,69 \%$ & $\mathrm{C}_{3} \mathrm{~A}$ & 6064725,29 & 6243172,24 & $2,94 \%$ \\
\hline $\mathrm{Al}_{2} \mathrm{O}_{3}$ & 2459361,43 & 2447970,84 & $0,46 \%$ & $(\mathrm{CaO})_{4}$ & 22041142,08 & 3153745,7 & $85,69 \%$ \\
\hline $\mathrm{Fe}_{2} \mathrm{O}_{3}$ & 1340304,84 & 1269307,79 & $5,30 \%$ & $\mathrm{Al}_{2} \mathrm{O}_{3}$ & 2447395,14 & 1810641,81 & $26,02 \%$ \\
\hline $\mathrm{MgO}$ & 820755,67 & 813046,38 & $0,94 \%$ & $\mathrm{Fe}_{2} \mathrm{O}_{3}$ & 2179805,72 & 2112828,09 & $3,07 \%$ \\
\hline $\mathrm{K}_{2} \mathrm{O}$ & 482780,17 & 360229,26 & $25,38 \%$ & $\mathrm{MgO}$ & 1120945,36 & 1116355,59 & $0,41 \%$ \\
\hline $\mathrm{SO}_{3}$ & 82451,37 & 82298,93 & $0,18 \%$ & $\mathrm{SO}_{3}$ & 141315,82 & 137439 & $2,74 \%$ \\
\hline $\mathrm{Na}_{2} \mathrm{O}$ & 104498,78 & 129140,88 & $23,58 \%$ & $\mathrm{~K}_{2} \mathrm{O}$ & 581374,34 & 517037,42 & $11,07 \%$ \\
\hline Total & 47567977,2 & 46605877,09 & $2,02 \%$ & $\mathrm{Na}_{2} \mathrm{O}$ & 246000,31 & 159588,61 & $35,13 \%$ \\
\hline \multicolumn{4}{|c|}{ Carvão (318 K) } & Total & 83265566,08 & 61560066,01 & $26,07 \%$ \\
\hline $\mathrm{C}$ & 15187,18 & 5104 & $66,39 \%$ & \multicolumn{4}{|c|}{ Poeira $(1320 \mathrm{~K})$} \\
\hline $\mathrm{S}$ & 982,64 & 179,56 & $81,73 \%$ & $\mathrm{CaO}$ & 4044505,93 & 4040359,73 & $0,10 \%$ \\
\hline $\mathrm{N}_{2}$ & 516,67 & 109,58 & $78,79 \%$ & $\mathrm{SiO}_{2}$ & 27894,05 & 25895,67 & $7,16 \%$ \\
\hline $\mathrm{H}_{2}$ & 16527,34 & 4156,71 & $74,85 \%$ & $\mathrm{Al}_{2} \mathrm{O}_{3}$ & 78358,17 & 57270,8 & $26,91 \%$ \\
\hline $\mathrm{O}_{2}$ & 2067,35 & 272,8 & $86,80 \%$ & $\mathrm{Fe}_{2} \mathrm{O}_{3}$ & 155909,23 & 150116,46 & $3,72 \%$ \\
\hline $\mathrm{H}_{2} \mathrm{O}$ & 4948,47 & 4323,36 & $12,63 \%$ & $\mathrm{MgO}$ & 295571,35 & 294108,24 & $0,50 \%$ \\
\hline $\mathrm{CaO}$ & 138,5 & 19,16 & $86,17 \%$ & $\mathrm{SO}_{3}$ & 41262,53 & 40052,73 & $2,93 \%$ \\
\hline $\mathrm{SiO}_{2}$ & 3561,51 & 270,33 & $92,41 \%$ & $\mathrm{~K}_{2} \mathrm{O}$ & 160180,8 & 137296,38 & $14,29 \%$ \\
\hline $\mathrm{Al}_{2} \mathrm{O}_{3}$ & 1492 & 153,54 & $89,71 \%$ & $\mathrm{Na}_{2} \mathrm{O}$ & 42414,27 & 41912,46 & $1,18 \%$ \\
\hline $\mathrm{Fe}_{2} \mathrm{O}_{3}$ & 892,53 & 92,12 & $89,68 \%$ & Total & 4846096,33 & 4787012,47 & $1,22 \%$ \\
\hline $\mathrm{MgO}$ & 258,86 & 19,9 & $92,31 \%$ & \multicolumn{4}{|c|}{ Gases de chaminé (1373 K) } \\
\hline $\mathrm{K}_{2} \mathrm{O}$ & 43,3 & 11,95 & $72,40 \%$ & $\mathrm{CO}_{2}$ & 30088733,78 & 28410124,28 & $5,58 \%$ \\
\hline $\mathrm{SO}_{3}$ & 249,36 & 28,9 & $88,41 \%$ & $\mathrm{CO}$ & 256417,74 & 241406,92 & $5,85 \%$ \\
\hline Total & 46865,71 & 14741,92 & $68,54 \%$ & $\mathrm{SO}_{2}$ & 394416,6 & 389179,28 & $1,33 \%$ \\
\hline \multicolumn{4}{|c|}{$\operatorname{Ar}(1373 \mathrm{~K})$} & $\mathrm{H}_{2} \mathrm{O}$ & 7900863,4 & 7887257,97 & $0,17 \%$ \\
\hline $\mathrm{O}_{2} *$ & 35342985,7 & 37321199,77 & $5,60 \%$ & $\mathrm{O}_{2} * *$ & 25366122,86 & 25168123,58 & $0,78 \%$ \\
\hline $\mathrm{N}_{2}^{*}$ & 125003748,3 & 132722433,6 & $6,17 \%$ & $\mathrm{~N}_{2} * *$ & 133155929,5 & 132839210,2 & $0,24 \%$ \\
\hline Total & 160346734 & 170043633,4 & $6,05 \%$ & Total & 197162483,9 & 194935302,2 & $1,13 \%$ \\
\hline \multicolumn{8}{|c|}{ Reações $^{\forall}$} \\
\hline $\begin{array}{c}\mathrm{C}(\text { para } \\
\left.\mathrm{CO}_{2}\right)\end{array}$ & 186284202,1 & 186125797,6 & $0,09 \%$ & \multirow{4}{*}{$\begin{array}{l}\text { Exergia } \\
\text { perdida }\end{array}$} & \multirow{4}{*}{157585277,1} & \multirow{4}{*}{196295244,6} & \multirow{4}{*}{$24,56 \%$} \\
\hline $\begin{array}{c}\mathrm{H}_{2}(\text { para } \\
\left.\mathrm{H}_{2} \mathrm{O}\right)\end{array}$ & 44955847,2 & 51140985,58 & $13,76 \%$ & & & & \\
\hline $\mathrm{S}\left(\right.$ para $\left.\mathrm{SO}_{2}\right)$ & 3657797,2 & 3646589,74 & $0,31 \%$ & & & & \\
\hline Total & 234897846,5 & 240913372,9 & $2,56 \%$ & & & & \\
\hline Somatório & 442859423,4 & 457577625,3 & $3,32 \%$ & Somatório & 442859423,4 & 457577625,3 & $3,32 \%$ \\
\hline
\end{tabular}


Tabela AVI - Exergia de entrada e saída para as simulações 1 e 2 por componente.

[Table AVI - Inlet and outlet exergy for simulations 1 and 2 by component.]

\begin{tabular}{|c|c|c|c|c|c|}
\hline Entrada & $\begin{array}{c}\text { Simulação } 1 \\
\dot{\mathrm{E}}^{\mathrm{PH}}(\mathrm{kJ} / \mathrm{h})\end{array}$ & $\begin{array}{c}\text { Simulação } 2 \\
\dot{\mathrm{E}}^{\mathrm{PH}}(\mathrm{kJ} / \mathrm{h})\end{array}$ & Saída & $\begin{array}{c}\text { Simulação } 1 \\
\dot{\mathrm{E}}^{\mathrm{PH}}(\mathrm{kJ} / \mathrm{h})\end{array}$ & $\begin{array}{c}\text { Simulação } 2 \\
\dot{\mathrm{E}}^{\mathrm{PH}}(\mathrm{kJ} / \mathrm{h})\end{array}$ \\
\hline \multicolumn{3}{|c|}{ Farinha $(1065 \mathrm{~K})$} & \multicolumn{3}{|c|}{ Clínquer (1423 K) } \\
\hline $\mathrm{CaO}$ & 24621797,15 & 14773078,29 & $\mathrm{C}_{3} \mathrm{~S}$ & 37380411,89 & 37380411,89 \\
\hline $\mathrm{CO}_{2}$ & 9016235,75 & - & $\mathrm{C}_{2} \mathrm{~S}$ & 8928845,66 & 8928845,66 \\
\hline $\mathrm{SiO}_{2}$ & 7865850,11 & 7865850,11 & $\mathrm{C}_{3} \mathrm{~A}$ & 6243172,24 & 6243172,24 \\
\hline $\mathrm{Al}_{2} \mathrm{O}_{3}$ & 2447970,84 & 2447970,84 & $(\mathrm{CaO})_{4}$ & 3153745,7 & 3153745,7 \\
\hline $\mathrm{Fe}_{2} \mathrm{O}_{3}$ & 1269307,79 & 1269307,79 & $\mathrm{Al}_{2} \mathrm{O}_{3}$ & 1810641,81 & 1810641,81 \\
\hline $\mathrm{MgO}$ & 813046,38 & 813046,38 & $\mathrm{Fe}_{2} \mathrm{O}_{3}$ & 2112828,09 & 2112828,09 \\
\hline $\mathrm{K}_{2} \mathrm{O}$ & 360229,26 & 360229,26 & $\mathrm{MgO}$ & 1116355,59 & 1116355,59 \\
\hline $\mathrm{SO}_{3}$ & 82298,93 & 82298,93 & $\mathrm{SO}_{3}$ & 137439 & 137439 \\
\hline $\mathrm{Na}_{2} \mathrm{O}$ & 129140,88 & 129140,88 & $\mathrm{~K}_{2} \mathrm{O}$ & 517037,42 & 517037,42 \\
\hline $\mathrm{CaCO}_{3}$ & - & 17664318,11 & $\mathrm{Na}_{2} \mathrm{O}$ & 159588,61 & 159588,61 \\
\hline Total & 46605877,09 & 45405240,59 & Total & 61560066,01 & 61560066,01 \\
\hline \multicolumn{3}{|c|}{ Carvão (318 K) } & \multicolumn{3}{|c|}{ Poeira (1320 K) } \\
\hline $\mathrm{C}$ & 5104 & 5104 & $\mathrm{CaO}$ & 4040359,73 & 4040359,73 \\
\hline $\mathrm{S}$ & 179,56 & 179,56 & $\mathrm{SiO}_{2}$ & 25895,67 & 25895,67 \\
\hline $\mathrm{N}_{2}$ & 109,58 & 109,58 & $\mathrm{Al}_{2} \mathrm{O}_{3}$ & 57270,8 & 57270,8 \\
\hline $\mathrm{H}_{2}$ & 4156,71 & 4156,71 & $\mathrm{Fe}_{2} \mathrm{O}_{3}$ & 150116,46 & 150116,46 \\
\hline $\mathrm{O}_{2}$ & 272,8 & 272,8 & $\mathrm{MgO}$ & 294108,24 & 294108,24 \\
\hline $\mathrm{H}_{2} \mathrm{O}$ & 4323,36 & 4323,36 & $\mathrm{SO}_{3}$ & 40052,73 & 40052,73 \\
\hline $\mathrm{CaO}$ & 19,16 & 19,16 & $\mathrm{~K}_{2} \mathrm{O}$ & 137296,38 & 137296,38 \\
\hline $\mathrm{SiO}_{2}$ & 270,33 & 270,33 & $\mathrm{Na}_{2} \mathrm{O}$ & 41912,46 & 41912,46 \\
\hline $\mathrm{Al}_{2} \mathrm{O}_{3}$ & 153,54 & 153,54 & Total & 4787012,47 & 4787012,47 \\
\hline $\mathrm{Fe}_{2} \mathrm{O}_{3}$ & 92,12 & 92,12 & \multicolumn{3}{|c|}{ Gases de chaminé (1373 K) } \\
\hline $\mathrm{MgO}$ & 19,9 & 19,9 & $\mathrm{CO}_{2}$ & 28410124,28 & 29234674,51 \\
\hline $\mathrm{K}_{2} \mathrm{O}$ & 11,95 & 11,95 & $\mathrm{CO}$ & 241406,92 & 241406,92 \\
\hline $\mathrm{SO}_{3}$ & 28,9 & 28,9 & $\mathrm{SO}_{2}$ & 389179,28 & 389179,28 \\
\hline \multirow[t]{2}{*}{ Total } & 14741,92 & 14741,92 & $\mathrm{H}_{2} \mathrm{O}$ & 7887257,97 & 7887257,97 \\
\hline & $\operatorname{Ar}(1373$ e $298 \mathrm{~K})$ & & $\mathrm{O}_{2} * *$ & 25168123,58 & 25168123,58 \\
\hline $\mathrm{O}_{2} *$ & 37321199,77 & 37321199,77 & $\mathrm{~N}_{2}^{* *}$ & 132839210,2 & 132839210,2 \\
\hline $\mathrm{N}_{2}^{*}$ & 132722433,6 & 132722433,6 & Total & 194935302,2 & 195759852,4 \\
\hline Total & 170043633,4 & 170043633,4 & \multicolumn{3}{|c|}{ Exergia de reação ${ }^{\forall}$} \\
\hline \multicolumn{3}{|c|}{ Exergia de reação ${ }^{\forall}$} & Formação $\mathrm{C}_{3} \mathrm{~S}$ & - & 19093056,67 \\
\hline $\mathrm{C}\left(\right.$ para $\left.\mathrm{CO}_{2}\right)$ & 186125797,6 & 181100401,1 & Formação $\mathrm{C}_{2} \mathrm{~S}$ & - & 7911644,144 \\
\hline $\mathrm{C}($ para $\mathrm{CO})$ & - & 2158936,55 & Formação $\mathrm{C}_{3} \mathrm{~A}$ & - & $-774111,36$ \\
\hline $\mathrm{H}_{2}\left(\right.$ para $\left.\mathrm{H}_{2} \mathrm{O}\right)$ & 51140985,58 & 51140985,58 & Formação $\mathrm{C}_{4} \mathrm{AF}$ & - & $-257610,705$ \\
\hline $\mathrm{S}\left(\right.$ para $\left.\mathrm{SO}_{2}\right)$ & 3646589,74 & 3646589,74 & Calcinação & - & $-64380006,54$ \\
\hline \multirow[t]{2}{*}{ Total } & 240913372,9 & 238046913 & Total & - & $-38407027,79$ \\
\hline & & & Exergia perdida & 196295244,6 & 229810625,7 \\
\hline Somatório & 457577625,3 & 453510528,8 & Somatório & 457577625,3 & 453510528,8 \\
\hline
\end{tabular}

* - ar primário + ar secundário; ${ }^{* *}$ - ar residual, ${ }^{\forall}$ - valores para Ex $(\mathrm{kJ} / \mathrm{h})$. 


\section{REFERÊNCIAS}

[1] Cembureau, "Key facts \& figures", https://cembureau. eu.

[2] S.A.A.H. Alyasiry, I.S. Alkroosh, P.K. Sarker, Case Stud. Constr. Mater. 7 (2017) 91.

[3] D.C.Q. Rodrigues, A.P. Soares Jr, E.F. Costa Jr, A.O.S. Costa, Cerâmica 59, 350 (2013) 302.

[4] S. Fellaou, T. Bounahmidi, Appl. Therm. Eng. 115 (2017) 1161.

[5] CSI, "Development of state of the art techniques in cement manufacturing: trying to look ahead", Eur. Cem. Res. Acad. (2017).

[6] D.C.Q. Rodrigues, A.P. Soares Jr, E.F. Costa Jr, A.O.S. Costa, An. Acad. Bras. Cienc. 89 (2017) 3123.

[7] H.M. Souza, A.P. Soares Jr, E.F. Costa Jr, A.O.S. Costa, Cerâmica 61, 357 (2015) 23.

[8] G. Kabir, A.I. Abubakar, U.A. El-Nafaty, Energy 35 (2010) 1237.

[9] A. Atmaca, R. Yumrutas, Appl. Therm. Eng. 66 (2014) 435.

[10] C.G. Manias, in: Innov. Portland Cem. Manuf., Eds. J.I. Bhatty, F.M. Miller, S.H. Kosmatka, Portland Cem. Ass. (2004).
[11] A. Ustaoglu, M. Alptekin, M.E. Akay, Appl. Therm. Eng. 112 (2017) 281.

[12] Ü. Çamdali, A. Eri'şen, F. Çelen, Energy Convers. Manag. 45 (2004) 3017.

[13] M.J. Moran, H.N. Shapiro, Princípios de termodinâmica para engenharia, LTC, Rio Janeiro (2013).

[14] Z. Sogut, Z.E. Oktay, Z. Karakoç, Appl. Therm. Eng. 30 (2010) 817.

[15] Nat. Inst. Stand. Tech., "Pesquisa para dados de espécies através da fórmula química", http://webbook.nist.gov.

[16] J.M. Smith, H.C. Van Ness, M.M. Abbott, Introdução à termodinâmica da engenharia química, vol. $1,5^{\mathrm{a}}$ ed., JC, Rio Janeiro (2000).

[17] F. El-Mahallawy, S.E.-D. Habik, Fundamentals and technology of combustion, Elsevier Sci., Kidlington (2002).

[18] J. Warnatz, U. Maas, R.W. Dibble, Combustion physical and chemical fundamentals, modeling and simulation, experiments, pollutant formation, $4^{\mathrm{a}}$ ed., Springer, Berlin (2006).

[19] J.M. Mollerup, M.L. Michelsen, Thermodynamic models: fundamental \& computational aspects, $2^{\text {nd }}$ ed., Tieline, Denmark (2007).

[20] D.K. Fidaros, C.A. Baxevanou, C.D. Dritselis, N.S. Vlachos, Powder Techn. 171, 2 (2007) 81.

(Rec. 22/12/2017, Rev. 20/02/2018, Ac. 17/04/2018) 